\title{
ON THE CHORDAL TRANSFORM OF HILBERT SPACE OPERATORS
}

\author{
OMAR HIRZALLAH \\ Department of Mathematics, Hashemite University, Zarqa, Jordan \\ e-mail:o.hirzal@hu.edu.jo \\ and FUAD KITTANEH \\ Department of Mathematics, University of Jordan, Amman, Jordan \\ e-mail: fkitt@ju.edu.jo
}

(Received 28 August, 2000; accepted 24 October 2000)

\begin{abstract}
Let $B(H)$ denote the $C^{*}$-algebra of all bounded linear operators on a separable Hilbert space $H$. For $A, B \in B(H)$, the chordal transform $f_{A, B}$, as an operator on $B(H)$, is defined by $f_{A, B}(X)=\left(\left|A^{*}\right|^{2}+I\right)^{-1 / 2} \delta_{A, B}(X)\left(|B|^{2}+I\right)^{-1 / 2}$, where $\delta_{A, B}$ is the generalized derivation defined on $B(H)$ by $\delta_{A, B}(X)=A X-X B$. Orthogonality of the range and the kernel of $f_{A, B}$, with respect to the unitarily invariant norms $\|\mid$.$\| , are discussed. It is shown that if A, B$ are self-adjoint, then \|\|$f_{A, B}(X)\left\||\leq \|||X|||\right.$ for all $X$. Related norm inequalities comparing $f_{A, B}$ and $\delta_{A, B}$ are also given.
\end{abstract}

2000 Mathematics Subject Classification. Primary 47A30, 47B10, 47B15, 47B20, 47B47, 47B49. Secondary 46B20.

1. Introduction. Let $B(H)$ denote the $C^{*}$-algebra of all bounded linear operators on a separable Hilbert space $H$. For operators $A, B \in B(H)$, the generalized derivation $\delta_{A, B}$, as an operator on $B(H)$, is defined by

$$
\delta_{A, B}(X)=A X-X B
$$

for all $X \in B(H)$. If $N, M \in B(H)$ are normal, then $\delta_{N, M}$ is called a generalized normal derivation. Also, for $A, B \in B(H)$, we define the chordal transform $f_{A, B}$, as an operator on $B(H)$, by

$$
f_{A, B}(X)=\left(\left|A^{*}\right|^{2}+I\right)^{-1 / 2} \delta_{A, B}(X)\left(|B|^{2}+I\right)^{-1 / 2}
$$

for all $X \in B(H)$. When $A=B$, we simply write $f_{A}$ for $f_{A, A}$. The chordal transform has some geometric properties that resembles those of the chordal distance. Recall that the chordal distance between any two complex numbers $a$ and $b$ is given by

$$
d(a, b)=\frac{|a-b|}{\sqrt{|a|^{2}+1} \sqrt{|b|^{2}+1}} .
$$


It is easy to see that

$$
d(a, b) \leq 1
$$

for all complex numbers $a$ and $b$ (see, e.g., [16, pp. 316-317]).

The orthogonality of the range and the kernel of certain derivations has been extensively studied by several authors (see, e.g., [1], [2], [5], [9], [10], [12], [13], and references therein).

In Section 2 of this paper, we investigate the related orthogonality results for the chordal transform. In Section 3, we establish norm comparison for the generalized derivation $\delta_{A, B}$ and the chordal transform $f_{A, B}$, which are closely related to certain gap formulas that are useful in perturbation theory of operators (see, e.g., [8], [11], [15], and [17]).

Some of these comparison results enable us to give norm inequalities that are considered as noncommutative versions of the inequality (4).

In addition to the usual operator norm $\|$.$\| , which is defined on all of B(H)$, we are interested in the general class of unitarily invariant (or symmetric) norms. Each unitarily invariant norm $\|||$.$\| is defined on a norm ideal C_{\||| .||}$associated with it. This ideal, which is a Banach space under the norm $\||| .||$, is contained in the ideal of compact operators. Every unitarily invariant norm satisfies the invariance property

$$
\|U A V\|\|=\| A\|\|
$$

for all $A \in C_{|||.|||}$and for all unitary operators $U, V \in B(H)$. It also satisfies the symmetry property

$$
\||B A C|\| \leq\||| A|\||| C\|
$$

for all $A \in C_{|||.| \mid}$and for all $B, C \in B(H)$.

For a compact operator $A \in B(H)$, let $s_{1}(A) \geq s_{2}(A) \geq \ldots \geq 0$ denote the singular values of $A$, i.e., the eigenvalues of $|A|=\left(A^{*} A\right)^{1 / 2}$. Every unitarily invariant norm of an operator is a symmetric gauge function of the singular values of that operator. For $1 \leq p \leq \infty$, the Schatten $p$-norms

$$
\|A\|_{p}=\left(\sum_{j=1}^{\infty} s_{j}^{p}(A)\right)^{1 / p}
$$

are typical examples of unitarily invariant norms, where by convention $\|A\|_{\infty}=s_{1}(A)$ is the usual operator norm of the compact operator $A$. The norm ideals associated with these norms are the Schatten $p$-classes $C_{p}, 1 \leq p \leq \infty$. Hence $C_{1}, C_{2}$, and $C_{\infty}$ are the trace class, the Hilbert-Schmidt class, and the class of compact operatos, respectively. The Hilbert-Schmidt class is a Hilbert space under the inner product

$$
\langle A, B\rangle=\operatorname{tr} B^{*} A=\operatorname{tr} A B^{*},
$$

where $t r$ is the trace functional. So the Hilbert-Schmidt norm is also given by

$$
\|A\|_{2}=\left(\operatorname{tr} A^{*} A\right)^{1 / 2}=\left(\sum_{i, j=1}^{\infty}\left|\left\langle A f_{j}, e_{i}\right\rangle\right|^{2}\right)^{1 / 2}
$$

where $\left\{e_{j}\right\}$ and $\left\{f_{j}\right\}$ are any orthonormal bases for $H$. For the theory of unitarily invariant norms, the reader is referred to [3], [7], or [18]. 
2. Orthogonality of the Range and the Kernel of $\boldsymbol{f}_{\boldsymbol{A}, \boldsymbol{B}}$. It has been shown in [10, Theorem 2] that if $N, M, S \in B(H)$ such that $N, M$ are normal, $S \in C_{2}$, and $N S=S M$, then

$$
\left\|\delta_{N, M}(X)+S\right\|_{2}^{2}=\left\|\delta_{N, M}(X)\right\|_{2}^{2}+\|S\|_{2}^{2}
$$

for all $X \in B(H)$. This says that, in the usual Hilbert space sense, $\operatorname{ran} \delta_{N, M} \cap C_{2}$ is orthogonal to ker $\delta_{N, M} \cap C_{2}$, where ran $\delta_{N, M}$ and ker $\delta_{N, M}$ denote the range and the kernel of $\delta_{N, M}$, respectively.

Moreover, it has been shown in [12, Corollary 1] that if $N, M, S \in B(H)$ such that $N, M$ are normal, $S \in C_{\||| .||}$, and $N S=S M$, then

$$
\left\|\left|\delta_{N, M}(X)+S\right|\right\||\geq\||S|\|
$$

for all $X \in B(H)$. That is, with respect to the unitarily invariant norm $\|||$.$\| , ran$ $\delta_{N, M} \cap C_{|||.| \mid}$is orthogonal, in the sense of [1, Definition 1.2], to $\operatorname{ker} \delta_{N, M} \cap C_{|||.| \mid}$. In this result and in the sequel, it is assumed that if $T \notin C_{|||.|||}$, then $\||| T|| \mid=\infty$. Generalizations of (10) and (11) to certain nonnormal derivations have been also given in [10] and [12], respectively. For the usual operator norm, (10) has been proved earlier by Anderson [1], and for the Schatten $p$-norms, it has been proved by Maher [14].

Our first orthogonality result for $f_{A, B}$ can be stated as follows.

Theorem 1. Let $N, S \in B(H)$ such that $N$ is normal, $S \in C_{2}$, and $N S=S N$. Then

$$
\left\|f_{N}(X)+S\right\|_{2}^{2}=\left\|f_{N}(X)\right\|_{2}^{2}+\|S\|_{2}^{2}
$$

for all $X \in B(H)$.

Proof. If $f_{N}(X)+S \notin C_{2}$, then $f_{N}(X) \notin C_{2}$; so $\left\|f_{N}(X)+S\right\|_{2}^{2}=\left\|f_{N}(X)\right\|_{2}^{2}+\|S\|_{2}^{2}=\infty$. Suppose that $f_{N}(X)+S \in C_{2}$. Then $f_{N}(X) \in C_{2}$, and so $S^{*} f_{N}(X) \in C_{1}$. Since $N$ is normal and $N S=S N$, it follows by the Fuglede theorem that $N^{*} S=S N^{*}$, and so $S^{*} N=N S^{*}$. Now

$$
\begin{aligned}
S^{*} f_{N}(X) & =S^{*}\left(\left|N^{*}\right|^{2}+I\right)^{-1 / 2}(N X-X N)\left(|N|^{2}+I\right)^{-1 / 2} \\
& =S^{*}\left(\left|N^{*}\right|^{2}+I\right)^{-1 / 2} N X\left(|N|^{2}+I\right)^{-1 / 2}-S^{*}\left(\left|N^{*}\right|^{2}+I\right)^{-1 / 2} X N\left(\left|N^{2}\right|+I\right)^{-1 / 2} \\
& =S^{*} N\left(\left|N^{*}\right|^{2}+I\right)^{-1 / 2} X\left(|N|^{2}+I\right)^{-1 / 2}-S^{*}\left(\left|N^{*}\right|^{2}+I\right)^{-1 / 2} X\left(|N|^{2}+I\right)^{-1 / 2} N \\
& =N S^{*}\left(\left|N^{*}\right|^{2}+I\right)^{-1 / 2} X\left(|N|^{2}+I\right)^{-1 / 2}-S^{*}\left(\left|N^{*}\right|^{2}+I\right)^{-1 / 2} X\left(|N|^{2}+I\right)^{-1 / 2} N \\
& =N Y-Y N,
\end{aligned}
$$

where $Y=S^{*}\left(\left|N^{*}\right|^{2}+I\right)^{-1 / 2} X\left(|N|^{2}+I\right)^{-1 / 2}$; so $Y \in C_{2}$ and $N Y-Y N \in C_{1}$. Employing a result of Weiss [20, Theorem 8], we get $\operatorname{tr} S^{*} f_{N}(X)=0$. Now

$$
\begin{aligned}
\left\|f_{N}(X)+S\right\|_{2}^{2} & =\left\|f_{N}(X)\right\|_{2}^{2}+\|S\|_{2}^{2}+2 \operatorname{Re} \operatorname{tr} S^{*} f_{N}(X) \\
& =\left\|f_{N}(X)\right\|_{2}^{2}+\|S\|_{2}^{2},
\end{aligned}
$$

as required 
Using an argument similar to that used in the proof of Theorem 2 in [10], Theorem 1 can be applied to yield the following corollary.

Corollary 1. Let $N, M, S \in B(H)$ such that $N, M$ are normal, $S \in C_{2}$, and $N S=S M$. Then

$$
\left\|f_{N, M}(X)+S\right\|_{2}^{2}=\left\|f_{N, M}(X)\right\|_{2}^{2}+\|S\|_{2}^{2}
$$

for all $X \in B(H)$.

Proof. On $H \oplus H$, let $L=\left[\begin{array}{cc}N & 0 \\ 0 & M\end{array}\right], T=\left[\begin{array}{ll}0 & S \\ 0 & 0\end{array}\right]$, and $Y=\left[\begin{array}{cc}0 & X \\ 0 & 0\end{array}\right]$. Then $L$ is normal, $\quad T \in C_{2}, \quad L T=T L, \quad f_{L}(Y)=\left[\begin{array}{cc}0 & f_{N, M}(X) \\ 0 & 0\end{array}\right], \quad$ and $\quad f_{L}(Y)+T=$ $\left[\begin{array}{cc}0 & f_{N, M}(X)+S \\ 0 & 0\end{array}\right]$. Now the result follows by applying Theorem 1 to the operators $L, T$, and $Y$.

It has been pointed out in [12] that (10) and (11) can be extended to derivations induced by any pair of operators $(A, B)$ that satisfies the Fuglede-Putnam property, i.e., $A^{*} S=S B^{*}$, whenever $A S=S B$, where $S \in B(H)$. For several such pairs, we refer to [4] and references therein. In the same spirit, and again by using an argument similar to that used in the proof of Theorem 2 in [10], Corollary 1 can be applied to yield the following more general Hilbert space orthogonality result for $f_{A, B}$.

Corollary 2. Let $A, B, S \in B(H)$ such that $(A, B)$ satisfies the Fuglede-Putnam property, $S \in C_{2}$, and $A S=S B$. Then

$$
\left\|f_{A, B}(X)+S\right\|_{2}^{2}=\left\|f_{A, B}(X)\right\|_{2}^{2}+\|S\|_{2}^{2}
$$

for all $X \in B(H)$

For the general class of unitarily invariant norms, one can employ the analysis in [12] to prove the following Banach space orthogonality result for $f_{A, B}$.

Theorem 2. Let $A, B, S \in B(H)$ such that $(A, B)$ satisfies the Fuglede-Putnam property, $S \in C_{|||.| \mid}$, and $A S=S B$. Then

$$
\left\|\left|f_{A, B}(X)+S\right|\right\| \geq \||| S||
$$

for all $X \in B(H)$.

It should be mentioned here that (14) and (15) remain also true if $A=B$ is a cyclic subnormal operator. For derivations, this has been proved in [10] and [12]. The example given in [12] shows that the cyclicity assumption on A cannot be dispensed with. 
For $A, B \in B(H)$ and $S \in C_{2}$, it can be shown that $\left\|\delta_{A, B}(X)+S\right\|_{2}^{2}=$ $\left\|\delta_{A, B}(X)\right\|_{2}^{2}+\|S\|_{2}^{2}$ for every $X \in C_{2}$ if and only if $S^{*} A=B S^{*}$. When $A=B$, this has been observed in [13]. In the same direction, we have the following characterization of those operators in $C_{2}$ which are orthogonal to $\operatorname{ran} f_{A, B} \mid C_{2}$.

Theorem 3. Let $A, B, S \in B(H)$ such that $S \in C_{2}$. Then

$$
\left\|f_{A, B}(X)+S\right\|_{2}^{2}=\left\|f_{A, B}(X)\right\|_{2}^{2}+\|S\|_{2}^{2}
$$

for all $X \in C_{2}$ if and only if $S_{1}^{*} A=B S_{1}^{*}$, where

$$
S_{1}=\left(\left|A^{*}\right|^{2}+I\right)^{-1 / 2} S\left(|B|^{2}+I\right)^{-1 / 2} .
$$

Proof. If $S_{1}^{*} A=B S_{1}^{*}$, then $\left(|B|^{2}+I\right)^{-1 / 2} S^{*}\left(\left|A^{*}\right|^{2}+I\right)^{-1 / 2} A=B\left(|B|^{2}+I\right)^{-1 / 2} S^{*}$ $\left(\left|A^{*}\right|^{2}+I\right)^{-1 / 2}$. This, together with the fact that $\operatorname{tr} Y Z=\operatorname{tr} Z Y$ whenever $Y Z, Z Y \in C_{1}$, implies that for every $X \in C_{2}$, we have

$$
\begin{aligned}
& \operatorname{tr} S^{*} f_{A, B}(X)=\operatorname{tr} S^{*}\left(\left|A^{*}\right|^{2}+I\right)^{-1 / 2}(A X-X B)\left(|B|^{2}+I\right)^{-1 / 2} \\
& =\operatorname{tr} S^{*}\left(\left|A^{*}\right|^{2}+I\right)^{-1 / 2} A X\left(|B|^{2}+I\right)^{-1 / 2}-\operatorname{tr} S^{*}\left(\left|A^{*}\right|^{2}+I\right)^{-1 / 2} X B\left(|B|^{2}+I\right)^{-1 / 2} \\
& =\operatorname{tr}\left(|B|^{2}+I\right)^{-1 / 2} S^{*}\left(\left|A^{*}\right|^{2}+I\right)^{-1 / 2} A X-\operatorname{tr}\left(|B|^{2}+I\right)^{-1 / 2} S^{*}\left(\left|A^{*}\right|^{2}+I\right)^{-1 / 2} X B \\
& =\operatorname{tr} B\left(|B|^{2}+I\right)^{-1 / 2} S^{*}\left(\left|A^{*}\right|^{2}+I\right)^{-1 / 2} X-\operatorname{tr}\left(|B|^{2}+I\right)^{-1 / 2} S^{*}\left(\left|A^{*}\right|^{2}+I\right)^{-1 / 2} X B \\
& =\operatorname{tr}\left(|B|^{2}+I\right)^{-1 / 2} S^{*}\left(\left|A^{*}\right|^{2}+I\right)^{-1 / 2} X B-\operatorname{tr}\left(|B|^{2}+I\right)^{-1 / 2} S^{*}\left(\left|A^{*}\right|^{2}+I\right)^{-1 / 2} X B \\
& =0 .
\end{aligned}
$$

Now

$$
\begin{aligned}
\left\|f_{A, B}(X)+S\right\|_{2}^{2} & =\left\|f_{A, B}(X)\right\|_{2}^{2}+\|S\|_{2}^{2}+2 \operatorname{Re} \operatorname{tr} S^{*} f_{A, B}(X) \\
& =\left\|f_{A, B}(X)\right\|_{2}^{2}+\|S\|_{2}^{2} .
\end{aligned}
$$

Conversely, if $\left\|f_{A, B}(X)+S\right\|_{2}^{2}=\left\|f_{A, B}(X)\right\|_{2}^{2}+\|S\|_{2}^{2}$ for every $X \in C_{2}$, then Retr $S^{*} f_{A, B}(X)=0$ for every $X \in C_{2}$. Replacing $X$ by $i X$, we get $\operatorname{Im} \operatorname{tr} S^{*} f_{A, B}(X)=0$, and so $\operatorname{tr} S^{*} f_{A, B}(X)=0$ for every $X \in C_{2}$. But by straightforward computations, we have $\operatorname{tr}\left(S_{1}^{*} A-B S_{1}^{*}\right) X=\operatorname{tr} S^{*} f_{A, B}(X)=0$ for every $X \in C_{2}$. Consequently, $S_{1}^{*} A-B S_{1}^{*}$ $=0$, and so $S_{1}^{*} A=B S_{1}^{*}$, as required.

Invoking the Gateaux differentiability of the Schatten p-norms and the usual operator norm, enables us to characterize those operators that are orthogonal to the rang of $f_{A, B}$ with respect to these norms. We leave the details to the interested reader. For derivations, related characterizations have been demonstrated in [5] and [13].

3. Norm Comparison Results for $\delta_{\boldsymbol{A}, \boldsymbol{B}}$ and $\boldsymbol{f}_{\boldsymbol{A}, \boldsymbol{B}}$. In this section we present several norm estimates comparing $\delta_{A, B}$ and $f_{A, B}$. Recall that if $T \in B(H)$ is self-adjoint, then the Cayley transform of $T$ is the unitary operator given by

$$
C(T)=(T-i I)(T+i I)^{-1}
$$


Our first comparison result can be stated as follows.

Theorem 4. Let $A, B \in B(H)$. Then

$$
\left\|\left|f_{A, B}(X)\|\| \leq\left\||| \delta_{A, B}(X)\right\|\right| \mid \leq\left(\|A\|^{2}+1\right)^{1 / 2}\left(\|B\|^{2}+1\right)^{1 / 2}\right\|\left\|f_{A, B}(X)\right\| \|
$$

for every $X \in B(H)$ and for every unitarily invariant norm $\|||$.$\| .$

Proof. Since $\left\|\left(|T|^{2}+I\right)^{-1 / 2}\right\| \leq 1 \quad$ and $\quad\left\|\left(|T|^{2}+I\right)^{1 / 2}\right\|=\left\|\left(\left|T^{*}\right|^{2}+1\right)^{1 / 2}\right\|=$ $\left(\|T\|^{2}+1\right)^{1 / 2}$ for every $T \in B(H)$, the desired inequalities follow from (2) and (6).

For the usual operator norm, the case $X=I$ of Theorem 4 is very closely related to Theorem 2.5 in [17], which asserts that the gap metric is equivalent to the metric generated by the usual operator norm (see also [11] and references therein).

For self-adjoint operators we have the following norm equality.

Theorem 5. Let $A, B \in B(H)$ be self-adjoint. Then

$$
2\left|\left\|f_{A, B}(X)\right\|\right|=\left\|\left|\delta_{C(A), C(B)}(X)\right|\right\| \mid
$$

for every $X \in B(H)$ and for every unitarily invariant norm $\|||$.$\| .$

Proof. The desired norm equality follows from the identity 2 if $_{A, B}(X)=\left(A^{2}+I\right)^{1 / 2}$ $(A-i I)^{-1} \delta_{C(A), C(B)}(X)(B-i I)^{-1}\left(B^{2}+I\right)^{1 / 2}$, the fact that $\left(T^{2}+I\right)^{1 / 2}(T-i I)^{-1}$ and $(T-i I)^{-1}\left(T^{2}+I\right)^{1 / 2}$ are unitary for every self-adjoint operator $T \in B(H)$, and the unitary invariance of $\|||$.

Combining (17) and (18) enables us to generalize a classical inequality of Fan and Hoffman [6] concerning Cayley transforms.

Corollary 3. Let $A, B \in B(H)$ be self-adjoint. Then

$$
\left\|\left|\delta_{C(A), C(B)}(X)\||| \leq 2 \mid\| \delta_{A, B}(X)\|\| \leq\left(\|\| A\|\|^{2}+1\right)^{1 / 2}\|B\|^{2}\right)^{1 / 2}|| \mid \delta_{C(A), C(B)}(X)\right\| \|
$$

for every $X \in B(H)$ and for every unitarily invariant norm ||$|| \mid$.

As another application of (18), we have the following noncommutative version of the inequality (4).

Corollary 4. Let $A, B \in B(H)$ be self-adjoint. Then

$$
\left\|\left|f_{A, B}(X)\||\leq\||| X \mid\|\right.\right.
$$

for every $X \in C_{\mid}\|$.$\| .$ 
Proof. Since $C(A)$ and $C(B)$ are unitary operators, it follows from (18) and the triangle inequality that

$$
\begin{aligned}
2\|\| f_{A, B}(X)\|\| & =\left\||| \delta_{C(A), C,(B)}(X)\right\| \| \\
& \leq\||| C(A) X|\|+\|| C(B) X|\|| \\
& =2|\|X \mid\|,
\end{aligned}
$$

from which the desired inequality follows.

It should be mentioned here that the inequality (20) remains also true if $A, B$ are assumed to be unitary operators. This follows from (2), the triangle inequality, and the unitary invariance of $\|||$.$\| .$

An extension of the inequality (20) to arbitary (i.e., not necessarily self-adjoint or unitary) operators is given in the following result, in which the direct sum $X \oplus Y$ stands for the $2 \times 2$ operator matrix $\left[\begin{array}{cc}X & 0 \\ 0 & Y\end{array}\right]$ defined on $H \oplus H$. Recall that if $\Phi$ is the symmetric gauge function associated with $\||| \mid$. , then

$$
\||X \oplus Y|\|=\Phi\left(s_{1}(X), s_{1}(Y), s_{2}(X), s_{2}(Y), \ldots\right)
$$

for all $X, Y \in C_{|||.| \mid .}$. Thus, in particular

$$
\|X \oplus Y\|=\max (\|X\|,\|Y\|)
$$

for all $X, Y \in B(H)$, and

$$
\|X \oplus Y\|_{p}=\left(\|X\|_{p}^{p}+\|Y\|_{p}^{p}\right)^{1 / p}
$$

for all $X, Y \in C_{p}$, where $1 \leq p<\infty$.

Corollary 5. Let $A, B \in B(H)$. Then

$$
\left\|\left|f_{A, B}(X) \oplus f_{A^{*}, B^{*}}(X)\right|\right\| \leq \||| X \oplus X|| \mid
$$

for every $X \in C_{\||| .||}$.

Proof. On $H \oplus H$, let $\tilde{A}=\left[\begin{array}{cc}0 & A \\ A^{*} & 0\end{array}\right], \tilde{B}=\left[\begin{array}{cc}0 & B^{*} \\ B & 0\end{array}\right]$, and $\tilde{X}=\left[\begin{array}{cc}0 & X \\ X & 0\end{array}\right]$. Now the inequality (24) follows by applying the inequality (20) to the self-adjoint operators $\tilde{A}, \tilde{B}$, and the operator $\tilde{X}$. Note that $\||\tilde{X}|\|=|\|X \oplus X \mid\|$ by the unitary invariance of ||l.|||.

Specializing the inequality (24) to the usual operator norm and the Schatten $p$-norms, we have the following corollary, which includes noncommutative versions of the inequality (4).

Corollary 6. Let $A, B \in B(H)$. Then

for every $X \in B(H)$.

$$
\text { (a) } \quad \max \left(\left\|f_{A, B}(X)\right\|,\left\|f_{A^{*}, B^{*}}(X)\right\|\right) \leq\|X\|
$$


(b)

$$
\left\|f_{A, B}(X)\right\|_{p}^{p}+\left\|f_{A^{*}, B^{*}}(X)\right\|_{p}^{p} \leq 2\|X\|_{p}^{p}
$$

for every $X \in C_{p}$, where $1 \leq p<\infty$. In particular, if $A, B$ are normal, then

$$
\left\|f_{A, B}(X)\right\|_{2} \leq\|X\|_{2}
$$

for every $X \in C_{2}$.

Proof. The inequalities (25) and (26) follow from the inequality (24), in view of the identities (22) and (23). The inequality (27) follows from (26) together with the fact that

$$
\left\|f_{A, B}(X)\right\|_{2}=\left\|f_{A^{*}, B^{*}}(X)\right\|_{2}
$$

for every $X \in B(H)$. To verify the identity (28), first we assume that $A, B$ are diagonal operators, say $A e_{j}=a_{j} e_{j}$ and $B f_{j}=b_{j} f_{j}$ for some orthonormal bases $\left\{e_{j}\right\}$ and $\left\{f_{j}\right\}$ of $\mathrm{H}$ and for some scalars $a_{j}, b_{j}, j=1,2, \ldots$. Then, by (9) and simple computations, we have

$$
\begin{aligned}
\left\|f_{A, B}(X)\right\|_{2}^{2} & =\sum_{i, j=1}^{\infty}\left|\left\langle f_{A, B}(X) f_{j}, e_{i}\right\rangle\right|^{2} \\
& =\sum_{i, j=1}^{\infty} \frac{\left|a_{i}-b_{j}\right|^{2}}{\left(\left|a_{i}\right|^{2}+1\right)\left(\left|b_{j}\right|^{2}+1\right)}\left|\left\langle X f_{j}, e_{i}\right\rangle\right|^{2} \\
& =\sum_{i, j=1}^{\infty} \frac{\left|\bar{a}_{i}-\bar{b}_{j}\right|^{2}}{\left(\left|a_{i}\right|^{2}+1\right)\left(\left|b_{j}\right|^{2}+1\right)}\left|\left\langle X f_{j}, e_{i}\right\rangle\right|^{2} \\
& =\left\|f_{A^{*}, B^{*}}(X)\right\|_{2}^{2} .
\end{aligned}
$$

Now for the general case (i.e., when $A, B$ are normal but not necessarily diagonal operators), we utilize the Voiculescu perturbation result [19], which says that every normal operator is the sum of a diagonal operator and a Hilbert-Schmidt operator with arbitrarily small Hilbert-Schmidt norm. This completes the proof of the corollary.

We remark here that an alternative proof of the inequality (27) can be based on the inequality (4), instead of the identity (28), together with the Voiculescu perturbation result mentioned above.

Our final comparison result is a considerable improvement of Theorem 4 for the Hilbert-Schmidt norm and for normal operators. In the proof of this result we need some orthogonality results given in Section 2.

Theorem 6. Let $A, B, S \in B(H)$ such that $A, B$ are normal, $S \in C_{2}$, and $A S=S B$. Then

$$
\left\|f_{A, B}(X)+S\right\|_{2} \leq\left\|\delta_{A, B}(X)+S\right\|_{2} \leq\left(\|A\|^{2}+1\right)^{1 / 2}\left(\|B\|^{2}+1\right)^{1 / 2}\left\|f_{A, B}(X)+S\right\|_{2}
$$

for every $X \in B(H)$. 
Proof. We have

$$
\begin{aligned}
& \left\|f_{A, B}(X)+S\right\|_{2}^{2}=\left\|f_{A, B}(X)\right\|_{2}^{2}+\|S\|_{2}^{2} \quad(\text { by Corollary 1) } \\
& \leq\left\|\delta_{A, B}(X)\right\|_{2}^{2}+\|S\|_{2}^{2} \quad \text { (by Theorem 4) } \\
& =\left\|\delta_{A, B}(X)+S\right\|_{2}^{2} \quad \text { (by the indentity (10)) }
\end{aligned}
$$

This proves the first inequality in (29).

To prove the second inequality in (29), let $S_{1}=\left(\left|A^{*}\right|^{2}+I\right)^{-1 / 2} S\left(|B|^{2}+I\right)^{-1 / 2}$. Then $S_{1} \in C_{2}$. Since $A S=S B$, it follows that $A S_{1}=S_{1} B$, and so

$$
\begin{aligned}
\left\|f_{A, B}(X)+S_{1}\right\|_{2}^{2} & =\left\|f_{A, B}(X)\right\|_{2}^{2}+\left\|S_{1}\right\|_{2}^{2} \quad \text { (by Corollary 1) } \\
& \left.\leq\left\|f_{A, B}(X)\right\|_{2}^{2}+\|S\|_{2}^{2} \quad \text { (by the inequality }(6)\right) \\
& =\left\|f_{A, B}(X)+S\right\|_{2}^{2} \quad \text { (by Corollary 1) }
\end{aligned}
$$

On the other hand

$$
\begin{aligned}
\left\|\delta_{A, B}(X)+S\right\|_{2} & =\left\|\left(\left|A^{*}\right|^{2}+I\right)^{1 / 2}\left(f_{A, B}(X)+S_{1}\right)\left(|B|^{2}+I\right)^{1 / 2}\right\|_{2} \\
& \leq\left(\|A\|^{2}+1\right)^{1 / 2}\left(\|B\|^{2}+1\right)^{1 / 2}\left\|f_{A, B}(X)+S_{1}\right\|_{2}
\end{aligned}
$$

(by the inequality (6))

Consequently,

$$
\left\|\delta_{A, B}(X)+S\right\|_{2} \leq\left(\|A\|^{2}+1\right)^{1 / 2}\left(\|B\|^{2}+1\right)^{1 / 2}\left\|f_{A, B}(X)+S\right\|_{2},
$$

which completes the proof of the theorem.

ACKNOWLEDGEments. The authors are grateful to the referee for his suggestions.

\section{REFERENCES}

1. J. H. Anderson, On normal derivations, Proc. Amer. Math. Soc. 38 (1973), 135-140.

2. M. Benlarbi Delai, S. Bouali and S. Cherki, Une remarque sur l'orthogonalité de l'image au noyau d'une dérivation générlisée, Proc. Amer. Math. Soc. 126 (1998), 167-171.

3. R. Bhatia. Matrix analysis (Springer-Verlag, 1997). $309-332$.

4. B. P. Duggal, On generalised Putnam-Fuglede theorems, Monatsh. Math. 107 (1989),

5. B. P. Duggal, Range kernel orthogonality of derivations, Linear Algebra Appl. 304 (2000), 103-108.

6. Ky Fan and A. J. Hoffman, Some metric inequalities in the space of matrices, Proc. Amer. Math. Soc. 6 (1955), 111-116.

7. I. C. Gohberg and M. G. Krein, Introduction to the theory of linear nonselfadjoint operators, Transl. Math. Monographs, Vol. 18 (Amer. Math. Soc., Providence, RI, 1969).

8. T. Kato, Perturbation theory of linear operators, 2nd ed. (Springer-Verlag, 1976).

9. D. Kečkić, Orthogonality of the range and kernel of some elementary operators, Proc. Amer. Math. Soc. 128 (2000), 3369-3377. 
10. F. Kittaneh, On normal derivations of Hilbert-Schmidt type, Glasgow Math. J. 29 (1987), 245-248.

11. F. Kittaneh, On some equivalent metrics for bounded operators on Hilbert space, Proc. Amer. Math. Soc. 110 (1990), 789-798.

12. F. Kittaneh, Normal derivations in norm ideals, Proc. Amer. Math. Soc. 123 (1995), $1779-1785$.

13. F. Kittaneh, Operators that are orthogonal to the range of a derivation, J. Math. Anal. Appl. 203 (1997), 868-873. 1000

14. P. J. Maher, Commutator approximants, Proc. Amer. Math. Soc. 115 (1992), 995-

15. A. McIntosh, Heinz inequalities and perturbation of spectral families, Macquarie Math. Reports, 1979.

16. D. S. Mitrinović, Analytic inequalities (Springer-Verlag, 1970).

17. R. Nakamoto, Gap formulas of operators and their applications, Math. Japon. 42 (1995), 219-232.

18. B. Simon, Trace ideals and their applications (Cambridge University Press, 1979).

19. D. Voiculescu, Some results on norm ideal perturbations of Hilbert space operators, J. Operator Theory 2 (1979), 3-37.

20. G. Weiss, The Fuglede commutativity theorem modulo the Hilbert-Schmidt class and generating functions for matrix operators I, Trans. Amer. Math. Soc. 246 (1978), 193-209. 